established. An interesting attempt was described to forecast by a model test using ultrasonics the performance at audible frequencies of an open-air theatre.

The widespread use of ultrasonics in biological investigation and in therapy is noteworthy. It appears that many hundreds of ultrasonic equipments for medical purposes costing $£ 100-£ 400$ apiece have been manufactured and are in use on the Continent of Europe. In contrast, it seems doubtful whether even a single equipment is used for this purpose in Great Britain. The total amount of equipment involved is probably comparable to that used for ultrasonic flaw detection throughout Europe, and the importance of this field is reflected in the fact that slightly more than 50 per cent of all the contributions to the Convention were on biology and medicine. At the exhibition of ultrasonic equipment which accompanied the Convention, it was noticeable that there was a great variety of therapeutic equipments preponderating over all other types of apparatus, even over that for flaw detection.

In biological applications the nature of the effect of ultrasonic radiation depends markedly on the intensity and on the frequency, which ranges from a few kilocycles to a few megacycles, and perhaps on whether pulsed radiation is employed. There appear to be broadly two effects, a heating effect and a mechanical effect. The former may differ markedly in its distribution from that when infra-red or radio short-wave radiation is used; for heat will be generated by the mechanical waves where absorption is most pronounced, while the depth of penetration will be less the higher the frequency employed. Thermal effects are more marked at high frequencies ; but mechanical damage more often occurs at low frequencies.

At lower intensities the mechanical effects of irradiation appear to be to cause acceleration of chemical reactions, liquefaction of thixotropic gels and an increased permeability of cell walls and membranes to the passage of natural fluids. These lower intensities are of the order of $\frac{1}{2}-3$ watts $/ \mathrm{sq} . \mathrm{cm}$. Above this level, prolonged irradiation causes irreversible changes and can break up the protein molecules themselves, as was shown by the investigations of several biological workers. Examples were cited of the irradiation of embryos, where the use of lower intensities accelerated development whereas higher intensity produced harmful and irreversible effects. The reaction of the nerve cells to radiation seems to involve a relaxation time, for it appears that the effect of stimulation continues even after the stimulus ceases. As a consequence, equipment has been developed which irradiates in successive bursts, the theory being that, while heating is reduced, the slowness of the physiological relaxation diminishes the effect of the gaps in transmission time, so favouring the mechanical effect in comparison with the heating effect.

It is impossible in this brief account to discuss adequately the thirty papers on medical aspects of the use of ultrasonics. Some forty maladies were listed which are believed to have benefited by irradiation. 'These range from treatment of abscesses, arthritis, asthma, ischias; carbuncles and lumbago to the treatment of neuralgia and spondylarthritis ankylopoietica Bechterew. In general, the intensity used is 1-5 watts/sq. cm., the treatment time varies from two to twelve minutes and the number of treatments from a few up to fourteen. Hundreds of treatments have been systematically examined, and there appears little doubt that benefit often results even in cases which have previously proved most obstinate to alternative treatments.

G. BradField

${ }^{1}$ Richardson, E. G., Nature, 164, 73 (1949).

2 Richardson, E. G., Nature, 164, 772 (1949)

${ }^{3}$ Bergmann, L., "Der Ultraschall"' (S. Hirzel Verlag, Stuttgart, 1949). ${ }^{4}$ Kê, T. S., Phys. Rev., 72, 41 (1947).

\section{ASTRONOMY IN AUSTRALIA}

\section{REPORT OF THE COMMONWEALTH ASTRON- OMER FOR 1949}

THE report of the Commonwealth Astrenomer for $1949^{*}$ covers the year up to December 31 and is divided into the following sections : solar investigation, time service, astrophysical observations, Melbourne catalogue, 74 -inch reflector, 48 -inch reflector, workshop, ionospheric prediction service, publications, staff and general. As very full descriptions are given under the different sections, it is impossible to do anything more than notice a few of the more outstanding features in the report.

Time service and astrophysical observations (the latter including seven sub-sections) occupy more than one-third of the report. During 1939 three additional frequency-standards were installed at the PostmasterGeneral's Research Laboratories, three at the National Standards Laboratory and three at Canberra. Improved methods of forecasting clock errors were introduced, and the radiated signals are now selfconsistent to within 2-3 milliseconds a day. Under the heading of astrophysical observations, it is satisfactory to learn that the spectrophotometric gradient observations of brighter stars were completed, and the results for 166 stars have been prepared for publication. The observations have provided increased precision to the blue end of the spectrumcolour relation, as some very blue southern stars were included, and this is important in the study of interstellar reddening and also of the stellar tempera. ture scale. Many will await with interest the publication of the results of the galactic radio emission at a frequency of $200 \mathrm{Mc} / \mathrm{s}$. on which observations have been made and corrections for aerial beam width applied.

Sir Howard Grubb, Parsons and Co. have the greater part of the heavy components of the 74-inch reflector ready for machining, and arrangements are being made for the contract for the supply and erection of the dome and building. The borosilicate glass disk for the 48-inch reflector has been cast by Messrs. Pilkington Bros., Ltd., and the optical work is in the hands of Messrs. Cox, Hargreaves and Thompson, Ltd. The Observatory workshop has been responsible for a number of major items, including : a three-prism slit spectrograph of dispersion $10 \mathrm{~A} . / \mathrm{mm}$. at $H \alpha$, which has been mounted in the Reynolds reflector; plate holders ; set of photometric sectors and mountings; and photometric calibrating equipment, constructed for the faint-star magnitude programme. Mirrors have been resilvered, and also adjustments and repairs made to various instruments submitted by Government organisations in Canberra.

* Commonwealth Observatory, Canberra. Report of the Commonwealth Astronomer for the year 1949. Pp. 6. (Canberra: Common wealth Government Printer, 1950.) 